\title{
High density mapping of atrial insertion of retrograde decremental accessory pathway 3D-illustration of accessory atrio-ventricular conduction network
}

Philippe Maury ${ }^{1}$, Quentin Voglimacci ${ }^{1}$, Franck Mandel ${ }^{1}$, Pauline Parlier ${ }^{2}$, Maud

Tabuteau $^{2}$, Maxime Beneyto ${ }^{1}$, and Anne Rollin ${ }^{1}$

${ }^{1}$ University Hospital Rangueil

${ }^{2}$ Boston Scientific Europe Headquarters

July 21, 2021

\begin{abstract}
We report the case of a reciprocating tachycardia using a purely retrograde decremental slow-conducting accessory pathway. High density 3D mapping of the atrial insertion reveals a large area of specific potentials at the level of the tricuspid annulus, which can be regarded as an accessory conduction network
\end{abstract}

High density mapping of atrial insertion of retrograde decremental accessory pathway

3D-illustration of accessory atrio-ventricular conduction network

Philippe Maury ${ }^{\text {a, b }}$, MD,

Quentin Voglimacci-Stephanopoli a, MD,

Franck Mandel a, MD,

Maud Tabuteau *, BE, Pauline Parlier*, BE

Maxime Beneyto a, MD, Anne Rollin ${ }^{\mathrm{a}}$, MD,

Division of Cardiology, University Hospital Rangueil Toulouse France

a. Department of Cardiology, University Hospital Rangueil, Toulouse, France

b. I2MC, INSERM UMR 1297, Toulouse, France

c. Boston Scientific, France

Financial disclosures: none (except for P Parlier and M Tabuteau who are employed by Boston Scientific)

Funding: none

Address for correspondance:

Dr. Philippe Maury, Cardiology, University Hospital Rangueil, 31059 Toulouse Cedex 09, France Tel: +33

(0) 561323054 Fax : + 33 (0) 561322246

e-mail: mauryjphil@hotmail.com

Key words: Mahaim fibers, decremental accessory pathway, high-density mapping, accessory atrio-ventricular node, ablation 
Abstract : we report the case of a reciprocating tachycardia using a purely retrograde decremental slowconducting accessory pathway. High density 3D mapping of the atrial insertion reveals a large area of specific potentials at the level of the tricuspid annulus, which can be regarded as an accessory conduction network.

Case :

We report the case of a 22 years old man with hypertrophic cardiomyopathy presenting with paroxysmal narrow QRS-supraventricular tachycardia. There was no preexcitation at baseline or during atrial pacing and no dual AV node physiology. Retrograde conduction was decremental and tachycardia was induced by ventricular extra-stimulus followed by an increase in VA interval and VAV pattern (fig 1).

Tachycardia displayed $1: 1$ AV relationship with relatively long VA interval (160 ms at the coronary sinus) and a VAV pattern after entrainment by ventricular fast pacing (fig 1). Ventricular extra-stimulus during tachycardia at the time the His bundle is refractory advanced the next atrial depolarization with decremental properties (fig 2). Tachycardia rate was slower when transient right bundle branch block was present (150 vs $170 \mathrm{bpm}$ ). Thus, reciprocating tachycardia utilizing a right bypass tract with unidirectional retrograde slow decremental conduction was diagnosed.

Mechanism was then more deeply investigated using ultra-high density 3D mapping system (Rhythmia , Boston Scientific, Inc). Earliest activation was mapped in the right atrium (shortest VA interval around 140 ms with large isolectrical interval), with a focal activation at the lateral part of the right atrium near the tricuspid annulus. A PPI equal to the tachycardia cycle length was elicited at this spot during entrainment. Interestingly, there was a potential following ventricular activation (around $80 \mathrm{~ms}$ after) in a relatively large area closer to the the annulus, which preceded the earliest atrial activation (around $60 \mathrm{~ms}$ before) (fig 2). Reannotating on this potential and then on successive atrial signals revealed a relatively wide structure at the level of the annulus, activated from the ventricle and then conducting to the atrium in a concentric fashion (fig 3). Ablation at the earliest atrial site did not terminate the tachycardia, but repeated ablation attempts on the expected location of the intermediate branching structure at the level of the annulus finally terminated the tachycardia which could never be induced again, and the patient remained free of any tachycardia at 6 months follow-up.

Discussion :

The tachycardia involved a concealed by-pass tract with relatively long and decremental retrograde conduction and without anterograde conduction (acting like purely retrograde « Mahaim » fibers). This extraanatomical retrograde conduction was associated with a particular wide structure located at the tricuspid annulus, harboring specific potential, and compatible with an accessory atrioventricular (AV) node and conduction pathway. To our knowledge, this is the first 3D documentation of such retrograde accessory pathway and of potential accessory AV node and conduction network.

Atrio-fascicular/ventricular connections (« Mahaim fibers $\gg$ ) usually do not demonstrate retrograde conduction $(1,2)$ and are considered to represent an accessory AV conduction pathway (2) because of decremental properties and of a specific potential - similar to His potential - recorded at the tricuspid annulus and further along the right lateral ventricular free wall $(1,2)$. It is expected to consist of a proximal component (similar to the AV node) at or above the tricuspid annulus, and a distal component (similar to the His bundle) that generates the specific potential, extending toward the right ventricular apex and distal components of the right bundle branch (2). Accessory AV node has been identified as an insulated tract of specialized cardiomyocytes, piercing the insulating pathways of the atrioventricular junction, and extending into the right ventricle, thus producing a second atrioventricular conduction system located on the lateral part of the tricuspid annulus, representing remnants of atrioventricular ring tissue (3). Atrial component of decremental accessory pathways, found in the vestibule of the tricuspid valve, could harbor remnants of the tissues that give rise to the normal AV node (4). Localization of the area of recording of a specific potential in our case 
was consistant with the location of the vestibule of the tricuspid valve (4) and thus could appear to be a 3D representation of what has been described in autopsies $(3,4)$. The relative wide area of recording was not indicative of common accessory pathway potentials, neither the long VA interval was evocative of conduction through an usual accessory pathway where local ventricular and atrial events are commonly fused. We were not able not depict the proximal (ventricular) end of the accessory pathway since it could not be mapped during orthodromic tachycardia (fused with global ventricular activation) and since there was no anterograde conduction.

3D mapping of decremental AP has been published but only for anterograde conduction (5-7). Rarely such decremental atrio-fascicular/ventricular connections demonstrated retrograde conduction (8-10). Concealed retrograde conduction had been demonstrated through the distal part of a decremental accessory pathway that blocked at its proximal atrial insertion (specific potential not followed by atrial activity) (11). Orthodromic reciprocating tachycardia utilizing such pathways have been sometimes documented $(9,10,12)$, but only in patients with anterograde conduction and pre-excitation. Thus, it seems to be the first report of orthodromic reciprocating tachycardia using a purely concealed decremental accessory pathway with findings compatible with an accessory AV node.

Some more common by-pass tracts however may also have decremental properties without being categorized as « Mahaim » fibers : these « short decrementally conducting AV pathways » may simply be due to alteration of conduction properties or tortuosity $(4,11)$. However they usually do not present with retrograde conduction $(2,12)$ and large areas of specific potentials are not described in this setting. Therefore, this case may be considered as a 3D illustration of orthodromic tachycardia involving an accessory AV node at the level of the tricuspid annulus in a patient without pre-excitation.

\section{References}

1. Ellenbogen KA, Vijayaraman P. Mahaim fibers: new electrophysiologic insights into an unusual variant. J Cardiovasc Electrophysiol. 2005;16:135-136

2. McClelland JH, Wang X, Beckman KJ, Hazlitt HA, Prior MI, Nakagawa H, Lazzara R, Jackman WM. Radiofrequency catheter ablation of right atriofascicular (Mahaim) accessory pathways guided by accessory pathway activation potentials. Circulation 1994;89:2655-66

3. Sternick E, Sánchez-Quintana D. Critical Assessment of the Concepts and Misconceptions of the Cardiac Conduction System over the Last 100 Years: The Personal Quest of Robert H. Anderson. J Cardiovasc Dev Dis. $2021 ; 8: 5$

4. Soares Correa F, Lokhandwala Y, Cruz Filho F, Sánchez-Quintana D, Mori S, Anderson RH, Wellens HJJ, Back Sternick E. Part II-Clinical presentation, electrophysiologic characteristics, and when and how to ablate atriofascicular pathways and long and short decrementally conducting accessory pathways. J Cardiovasc Electrophysiol. 2019;30:3079-3096

5. Fung JWH, Chan HCK, Chan WWL, Sanderson JE. Ablation of the Mahaim pathway guided by noncontact mapping. J Cardiovasc Electrophysiol. 2002;13:1064

6. Ortega M, Castrejón Castrejón S, Luis Merino J, Gutiérrez-Larraya F. Mahaim-type accessory pathway and right bundle branch electroanatomic delineation. Heart Rhythm Case Rep. 2015;1:266-267

7. Gupta A, Hsia HH, Lo R, Zei PC. Electroanatomic localization of a slowly conducting atrioventricular (Mahaim) accessory pathway. J Interv Card Electrophysiol2011;31:119-124

8. Kreiner G, Heinz G, Frey B, Gössinger HD. Demonstration of retrograde conduction over an atriofascicular accessory pathway.. J Cardiovasc Electrophysiol. 1997;8:74-9

9. Kalbfleisch S, Bowman K, Augostini R. A single Mahaim fiber causing both antidromic and orthodromic reciprocating tachycardia. J Cardiovasc Electrophysiol. 2008;19:740-2 
10. Vijayaraman P. Minimally decremental atriofascicular accessory pathway with bidirectional conduction. J Cardiovasc Electrophysiol. 2021;32:1782-1786

11. Haïssaguerre M, Cauchemez B, Marcus F, Le Métayer P, Lauribe P, Poquet F, Gencel L, Clémenty J. Characteristics of the ventricular insertion sites of accessory pathways with anterograde decremental conduction properties. Circulation. 1995;91:1077-85

12. De Ponti R, Storti C, Stanke A, Ferrari AA, Longobardi M, Salerno-Uriarte JA. Radiofrequency catheter ablation in patients with Mahaim-type slow-conduction accessory right atrioventricular pathway. Cardiologia 1994;39:169-80

Legends to figures

Figure 1 : Upper : induction of the reciprocating tachycardia by ventricular extra-stimulus with decremental retrograde conduction and a VAV pattern. Lower entrainment of the reciprocating tachycardia by fast ventricular pacing with a VAV pattern after the last ventricular paced beat $(\mathrm{S}=$ pacing artifact, $\mathrm{CS}$ and His = coronary sinus and His bundle electrodes, $\mathrm{A}=$ atrial electrogram, $\mathrm{H}=$ His electrogram).

Figure 2 : Upper : ventricular extra-stimulus delivered at the time the His bundle is refractory advanced the next atrial depolarization. Retrograde conduction through the accessory pathway has decremental properties, since although atrial event is advanced, VA interval was larger for shorter coupling interval of the premature beat. Lower : recording by the ablation catheter at the lateral tricuspid annulus : there is a specific potential between the ventricular (V) and atrial (A) electrograms during the tachycardia, similar to a His bundle potential

Figure 3 : high density 3D mapping of the right lateral part of the heart during the tachycardia, showing first the ventricular activation (blue) preceding the area of activation of the specific potential (violet) and then the atrial activation (red and yellow) with a concentric propagation from the preceding area. Besides are shown the potentials which were recorded by the system at each site (white $=$ ECG and atrial reference, red $=$ local bipolar electrogram, yellow $=$ local unipolar electrograms, green $=\ll$ trend $\gg$ in local activation as a summation of local electrograms). Of note that there was an additional component between the ventricular and atrial components on the trend. White dots represent the expected location of the tricuspid annulus, and black dots the approximate location of the additional structure responsible for the specific potential on the vestibular part of the valve, and which could be considered as an accessory AV conduction system/network. 

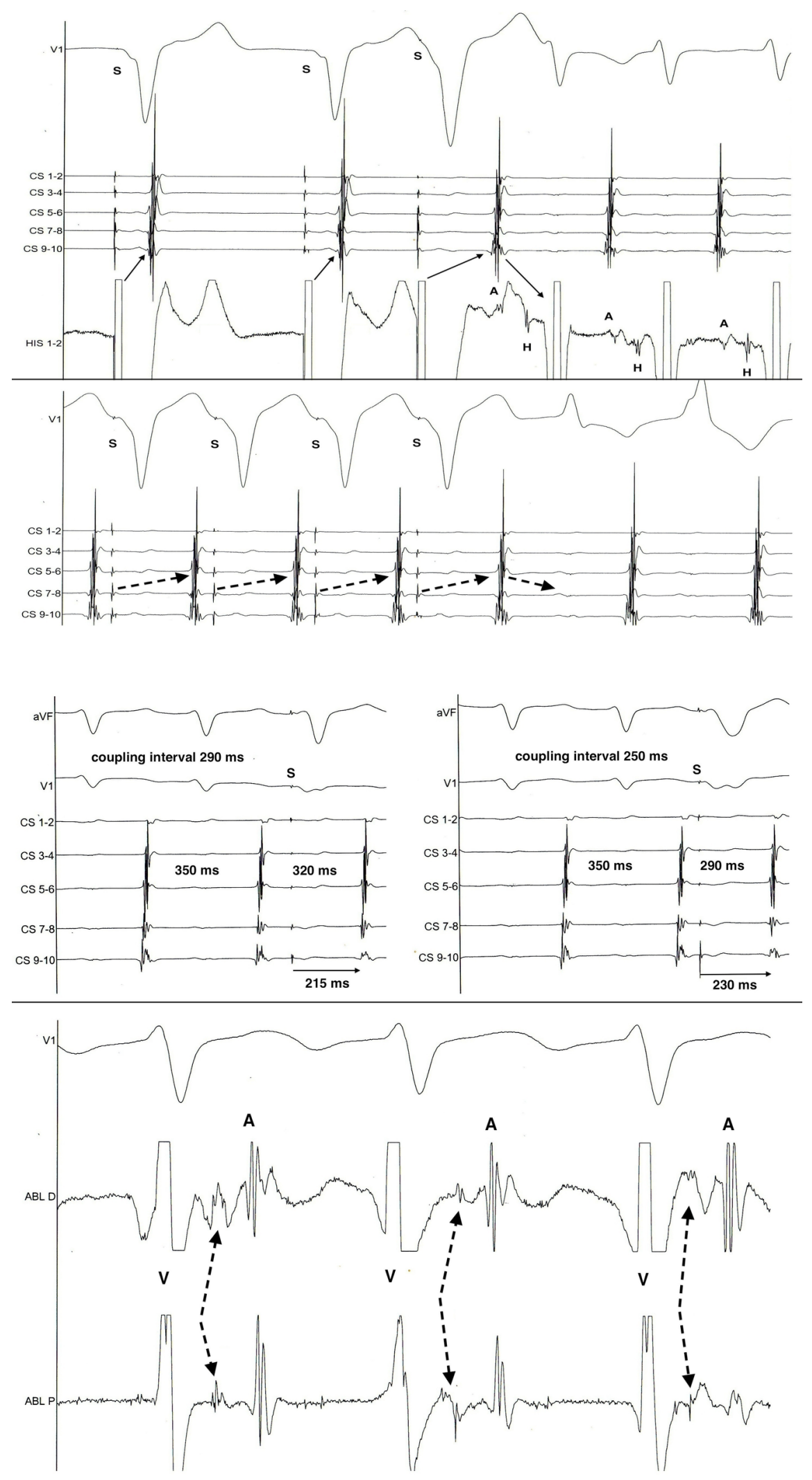


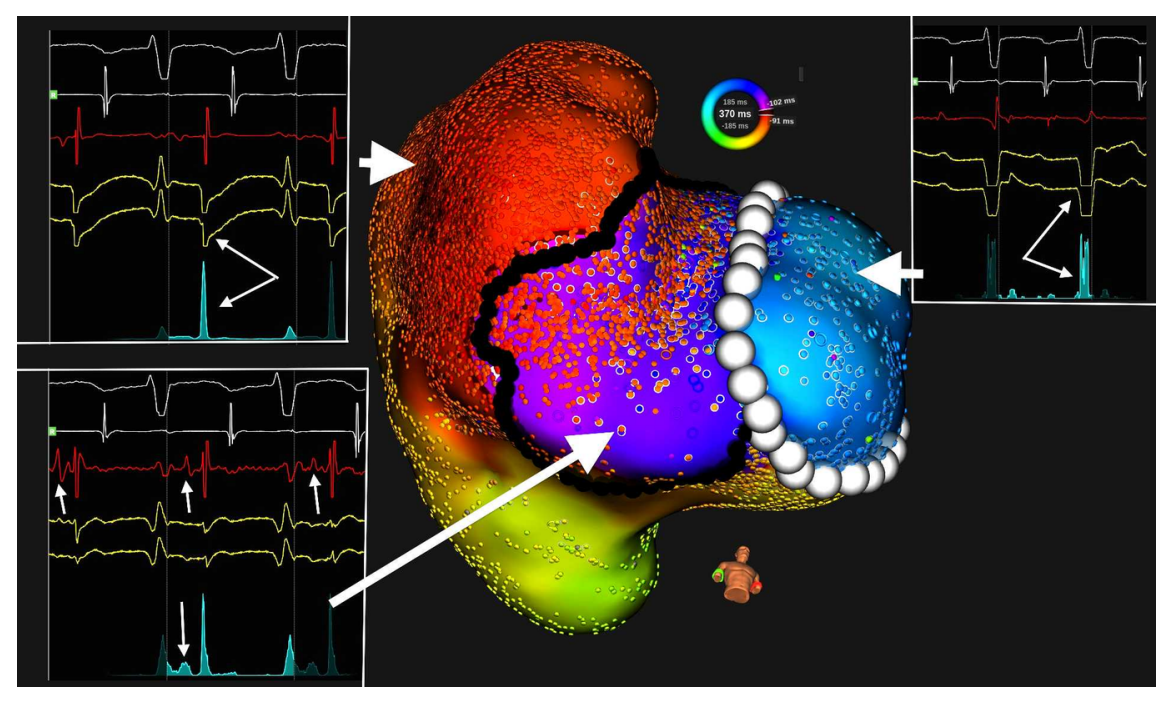

